\title{
Optimized Protocol for Linear RNA Amplification and Application to Gene Expression Profiling of Human Renal Biopsies
}

BioTechniques 34:546-556 (March 2003)

\section{A. Scherer, A. Krause, J.R. Walker', S.E. Sutton ${ }^{1}$, D. Serón ${ }^{2}$, F. Raulf, and M.P. Cooke \\ Novartis Pharma AG, Basel,} Switzerland; ${ }^{1}$ The Genomics Institute of the Novartis Research Foundation, San Diego, CA, USA; and ${ }^{2}$ Hospital de Bellvitge, Barcelona, Spain

\section{ABSTRACT}

Gene expression analysis using highdensity cDNA or oligonucleotide arrays is a rapidly emerging tool for transcriptomics, the analysis of the transcriptional state of a cell or organ. One of the limitations of current methodologies is the requirement of a relatively large amount of total or polyadenylated RNA as starting material. Standard array hybridization protocols require 5-15 $\mu \mathrm{g}$ labeled RNA. To obtain these quantities from small amounts of starting $R N A$ material, RNA can be amplified in a linear fashion. Here we introduce an optimized protocol for rapid and easy-to-use amplification of as little as $1 \mathrm{ng}$ total RNA. Our analysis shows that this method is linear and highly reproducible and that it preserves similarities as well as dissimilarities between normal and disease-related samples. We applied this technique to the RNA expression profiling of human renal allograft biopsies with normal histology and compared them to the profiles of renal biopsies with histological evidence of chronic transplant nephropathy or chronic rejection. Among others, complement component $\mathrm{Clr}$ was found to be significantly up-regulated in chronic rejection and chronic transplant nephropathy biopsies compared to normal samples, while fructose-1,6-biphosphatase showed lower-than-normal expression.

\section{INTRODUCTION}

Gene expression profiling with cDNA or oligonucleotide arrays has become a powerful tool for the investigation of the transcriptional state of tissues, cells, or subcellular fractions and has gained a huge impact on oncology, developmental biology, cell biology, and drug discovery research (1-9). Standard protocols for these studies require 5-15 $\mu \mathrm{g}$ labeled RNA (10), an amount that is difficult to obtain in some experimental designs and impossible in others, depending on the type and size of the tissue or sample $(6,11)$.

RNA amplification provides a solution to this problem. Currently, the two commonly used RNA amplification procedures are PCR (12) and amplified antisense RNA (aRNA) technology (13). During PCR, highly expressed genes or shorter transcripts may be amplified preferentially (14), which may lead to a strong overrepresentation of some transcripts, thus not reflecting reality.

The amplified aRNA procedure, a T7 RNA polymerase-based amplification of total RNA starting from 1 to 10 $\mu \mathrm{g}$, was first described by the Eberwine laboratory in 1990 (13) and has become a standard labeling procedure for GeneChip ${ }^{\circledR}$ technology (Affymetrix, Santa Clara, CA, USA) (10). This method is currently preferred over other RNA amplification protocols because RNA polymerase activity is generally not affected by the concentration of templates in a complex transcript mixture or by template sequences. Briefly, the Eberwine protocol employs the annealing of a synthetic oligo(dT) ${ }_{24} / \mathrm{T} 7 \mathrm{RNA}$ polymerase promoter primer. Following cDNA synthesis, RNA is synthesized by the addition of T7 RNA poly- merase. Additional rounds of amplification lower the amount of input RNA and have been reported to provide reproducible results (2).

Recently, a number of publications (15-17) reported salient quantitative analyses of gene expression profiles obtained from RNA amplification methods, all based on the procedure established by Eberwine. Although these methods gave highly reproducible results, the individual procedures and sample handling are rather labor intensive and therefore applicable only to a small number of samples at a time.

To overcome these limitations, we have modified the Eberwine method in a way that it now involves easy-to-use purification kits and standard laboratory settings. To assess the quality and reproducibility of the procedure, we amplified 1- $\mu \mathrm{g}, 100-\mathrm{ng}$, and 1-ng aliquots from total RNA of human peripheral blood mononuclear cells (PBMCs) and human kidney (HK) RNA and profiled them across 12600 probe sets representing about 12000 genes. Here we describe the RNA amplification method and compare microarray RNA expression data that were obtained from labeled aRNA after one, two, and three rounds of amplification (for $1 \mu \mathrm{g}, 100$ ng, and $1 \mathrm{ng}$, respectively).

Our data demonstrate the linearity and high reproducibility of the method. Additional support for these data was obtained from expression profiling experiments of human renal biopsies with normal histology and with histological evidence of chronic transplant nephropathy (CTN) or chronic rejection (CR). We demonstrate that our method preserves dissimilarities between these clinical samples, leading to the identification of genes with significant differential expression in CR and CTN. 


\section{MATERIALS AND METHODS}

\section{RNA Extraction}

Human PBMCs were purchased from Allcells (Oakland, CA, USA). Cells were subjected to RNA purification with RNeasy ${ }^{\circledR}$ (Qiagen, Valencia, CA, USA). The RNA was eluted with $80 \mu \mathrm{L}$ RNase-free water (Ambion, Austin, TX, USA). The total yield was $20 \mu \mathrm{g}$ total RNA per $1 \times 10^{7}$ cells, as measured spectrophotometrically (Beckman DU-600; Beckman Coulter, Fullerton, CA, USA). To standardize the RNA quality, HK total RNA (BD Biosciences Clontech, Palo Alto, CA, USA) was subjected to an RNeasy Cleanup procedure (Qiagen) according to the manufacturer's instructions. The quality of the RNA from both samples was tested by an Agilent 2100 Bioanalyzer (Agilent Technologies, Palo Alto, CA, USA).

\section{Biopsy RNA}

Total RNA of human renal protocol biopsies was provided by the Nephrology Department, Hospital de Bellvitge (Barcelona, Spain). All biopsy RNA extractions had been performed with RNeasy kit. Biopsies were performed and partly processed for routine light microscopy as previously described (18). Histological grading of the specimen was performed according to BANFF criteria (19).
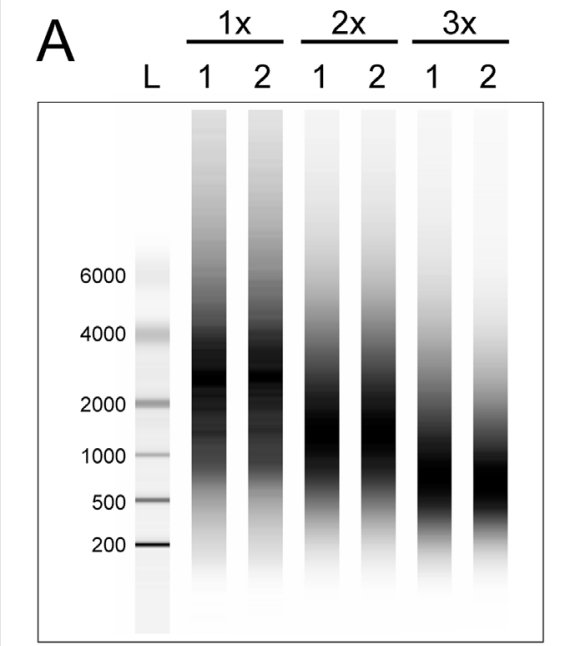

PBMC aRNA

C

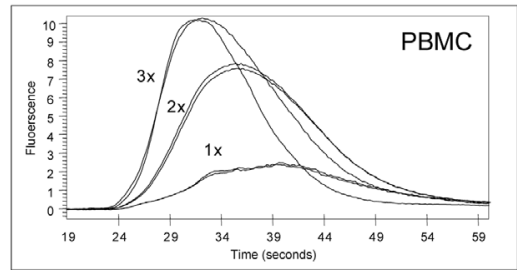

B

$\frac{1 \mathrm{x}}{12} \frac{2 \mathrm{x}}{12} \frac{3 \mathrm{x}}{12}$

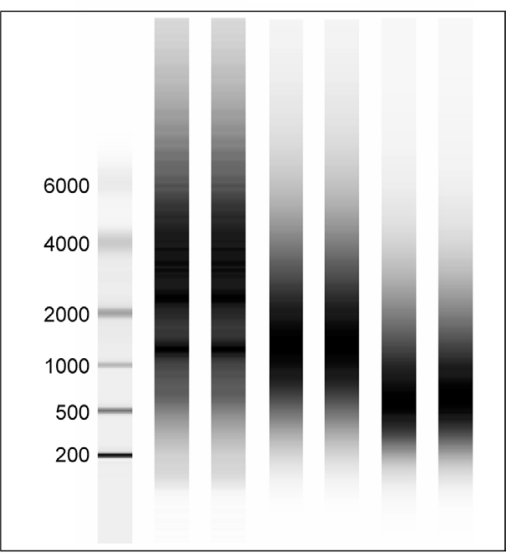

Human Kidney aRNA

D

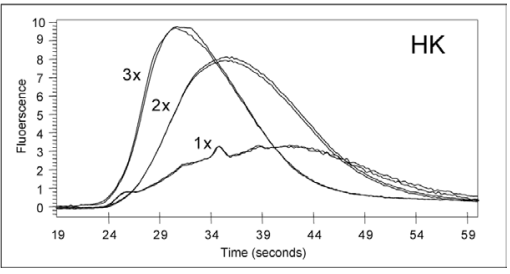

Figure 1. Effect of amplification on the size distribution of aRNA. Total RNA from PBMCs (A) or HK cells (B) was amplified in duplicate one, two, or three times, starting from $1 \mu \mathrm{g}, 100 \mathrm{ng}$, and $1 \mathrm{ng}$ total RNA, respectively. Biotinylated aRNA was tested for quality with an Agilent 2100 Bioanalyzer. The average size of the aRNA pool decreased with each round of amplification. L, RNA size marker in bp; lanes 1 and 2, duplicate aRNA of single $(1 \times)$, double $(2 \times)$, and triple $(3 \times)$ amplification reactions. (C and D) Electropherograms of the runs in panels A and B illustrate the decrease of the average size distribution of the aRNA pool. The scale on the horizontal axis is a measure for the time that expires from the start of the gel run until the detection of RNA by the detector. 


\section{Research Report}

\section{RNA Amplification and Microarray Processing}

All enzymes and buffers for the amplification procedure were purchased from Invitrogen (Carlsbad, CA, USA) unless explicitly mentioned. In the initial annealing reaction total RNA was incubated with 10 pmol T7-polydT primer [5'-GGCCAGTGAATTGTAATACGACTCACTATAGGGAGGCG$\mathrm{G}(\mathrm{T})_{24} \mathbf{3}^{\prime}$ ] (Genset SA, Paris, France) in a volume of $11 \mu \mathrm{L}$ at $70^{\circ} \mathrm{C}$ for $10 \mathrm{~min}$ and then at $42^{\circ} \mathrm{C}$ for $5 \mathrm{~min}$. The firststrand reaction was carried out in a volume of $20 \mu \mathrm{L}$ by the addition of $200 \mathrm{U}$ SuperScript II ${ }^{\mathrm{TM}}$ in the presence of firststrand buffer, $10 \mathrm{mM}$ DTT, $0.5 \mathrm{mM}$ dNTP mixture, and $1 \mu \mathrm{L}$ RNase inhibitor (Ambion) with a $42^{\circ} \mathrm{C}$ incubation for $1 \mathrm{~h}$. The second-strand synthesis was performed in a volume of 150 $\mu \mathrm{L}$ with $40 \mathrm{U} E$. coli DNA polymerase $\mathrm{I}$ in $1 \times$ second-strand buffer, $0.2 \mathrm{mM}$ dNTPs, $10 \mathrm{U}$ E. coli DNA ligase and 2 U RNase H. After a 2-h incubation at $16^{\circ} \mathrm{C}$, the dsDNA was blunted by the addition of $8 \mathrm{U}$ T4 DNA polymerase for $10 \mathrm{~min}$ at $16^{\circ} \mathrm{C}$. The dsDNA product was purified with a QIAquick ${ }^{\mathrm{TM}}$ PCR purification kit (Qiagen) and eluted in $50 \mu \mathrm{L}$ elution buffer. For only one round of amplification, the volume of the eluate was reduced to dryness under vacuum, resuspended in $22 \mu \mathrm{L}$ nuclease-free water, and then used in the RNA labeling reaction as described below. For additional rounds of amplification, the eluate was reduced to dryness under vacuum, resuspended in $8 \mu \mathrm{L}$ nuclease-free water, and subjected to an in vitro transcription reaction with the MEGAscript ${ }^{\mathrm{TM}}$ kit (Ambion), following the manufacturer's instructions for a 20 $\mu \mathrm{L}$ reaction volume. After a 3 -h incubation at $37^{\circ} \mathrm{C}$, the RNA was purified with the RNeasy kit system. The RNA was eluted in $30 \mu \mathrm{L}$ RNase-free water, reduced to dryness under vacuum, and resuspended in $11 \mu \mathrm{L}$ nuclease-free water.

The second round of RNA amplification varied in a few points slightly from the procedure described above. It employed the addition of $1 \mu \mathrm{L} 0.1$ $\mathrm{mg} / \mathrm{mL}$ random hexamer primers, followed by a 10 -min incubation at $70^{\circ} \mathrm{C}$. The reaction mixture was chilled on ice and then incubated at room temperature for $10 \mathrm{~min}$, at which point the first- strand synthesis reaction was started by the addition of $200 \mathrm{U}$ SuperScript II, 20 U RNase Inhibitor, $0.5 \mathrm{mM}$ dNTPs, and $10 \mathrm{mM}$ DTT in the presence of firststrand reaction buffer. The mixture was incubated at $37^{\circ} \mathrm{C}$ for $1 \mathrm{~h}$. A 20-min RNase $\mathrm{H}$ treatment $(2 \mathrm{U})$ at $37^{\circ} \mathrm{C}$ led to the degradation of the residual RNA. RNase $\mathrm{H}$ was heat-inactivated at $94^{\circ} \mathrm{C}$ for $2 \mathrm{~min}$, and the mixture was chilled on ice. The second-strand synthesis was initiated by the addition of $100 \mathrm{pmol}$ T7-polydT primer (see above) and incubation at $70^{\circ} \mathrm{C}$ for $5 \mathrm{~min}$, followed by $42^{\circ} \mathrm{C}$ for $10 \mathrm{~min}$. The second-strand synthesis was performed as described above, and the cDNA was purified with the QIAquick PCR purification kit. If this was the final round of amplification, then the volume of the eluate was reduced to dryness under vacuum and resuspended in $22 \mu \mathrm{L}$ nuclease-free water, followed by an in vitro RNA labeling procedure (see below).

If an additional (third) round of amplification was desired, then the eluate was reduced to dryness under vacuum and resuspended in $8 \mu \mathrm{L}$ nuclease-free water before it was then subjected to the procedure identical to that of a second round of amplification. We observed a 15 - to 30 -fold increase in aRNA in each round of amplification, resulting in $5 \times 10^{5}$ - to $1.9 \times 10^{6}$-fold amplification of mRNA after three rounds [assuming 3.3\% poly $(\mathrm{A})+$ RNA within the initial pool of total RNA]. Labeled RNAs were fractionated at $94^{\circ} \mathrm{C}$ for 35,25 , or $20 \mathrm{~min}$ for single, double, or triple amplified

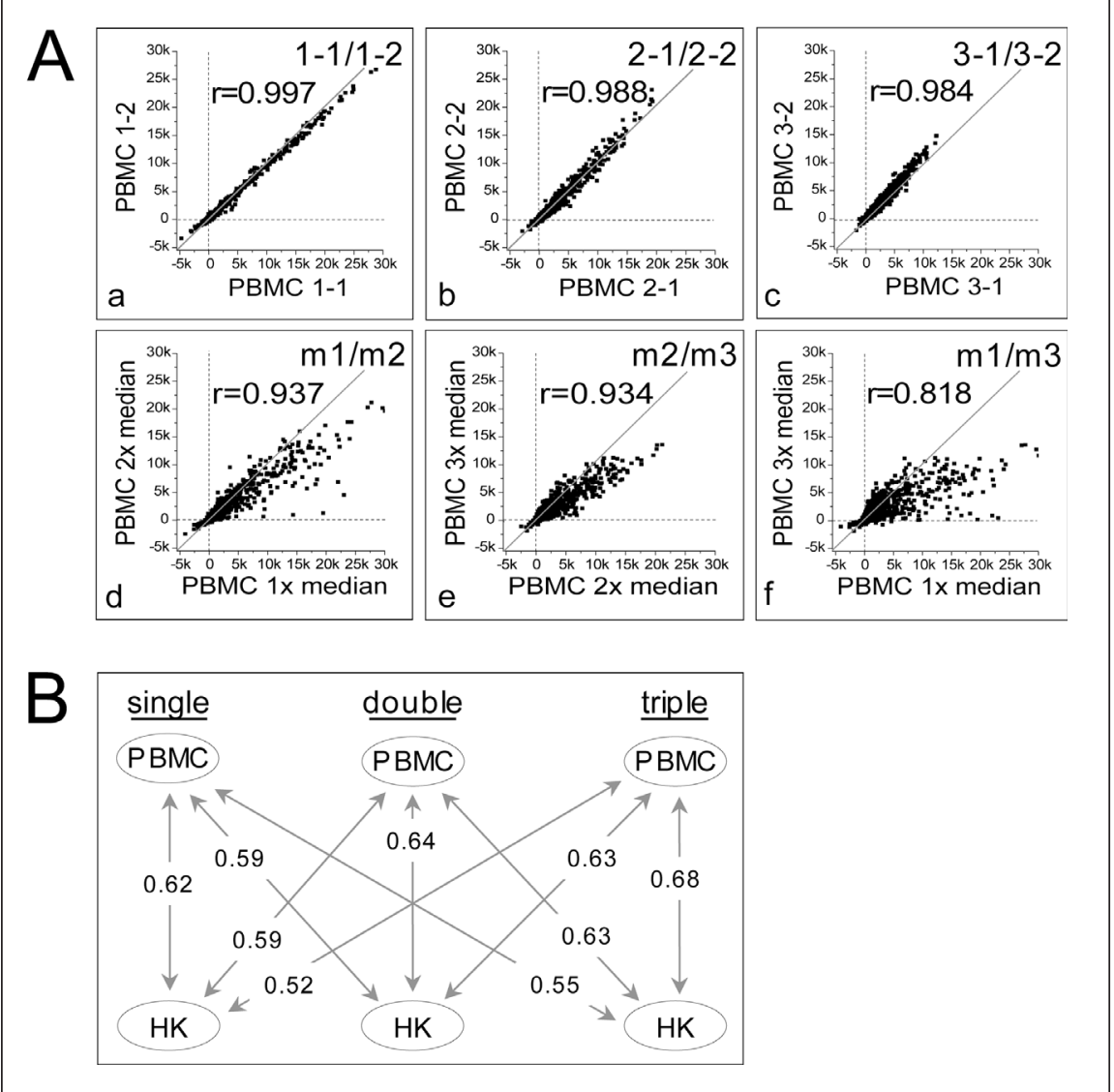

Figure 2. Assessment of the reproducibility of multiple rounds of RNA amplification by Pearson correlation. The correlation coefficient $r$ for duplicates are shown in a, b, and c (single, double, and triple amplification, respectively). Notation is as described in the text. (d, e, and f) Comparison of the median $\mathrm{m}$ of the duplicates through three rounds of amplification. Correlation coefficients close to 1.0 indicate that the amplification method is highly reproducible. (B) Comparison of gene expression signatures of of PBMC and HK by Pearson correlation. Correlation coefficent $r$ of the RNA expression values was calculated for the median $\mathrm{m}$ of the duplicate groups. The inter-group differences are conserved after three rounds of amplification. 


\section{Research Report}

RNAs, respectively. Shorter incubation times for double RNA and triple RNA were chosen to avoid complete degradation of the RNA.

The RNA biotinylation step was performed with the High-Yield ${ }^{\mathrm{TM}}$ RNA Labeling Kit (Enzo Diagnostics, Farmingdale, NY, USA) following the manufacturer's instructions. The labeled RNA was purified using an RNeasy kit. The RNA was eluted in $60 \mu \mathrm{L}$ RNase-free water and quantified by absorbance at $260 \mathrm{~nm}$. Labeled aRNA (15 $\mu \mathrm{g})$ was fractionated and hybridized; hybridization and staining were performed according to the Affymetrix Expression Analysis Technical Manual (10).

\section{Data Analysis}

Statistical analysis was done with SPlus ${ }^{\circledR}$, Version 6.0, by Insightful (Seattle, WA, USA). All analyses except where mentioned in the text were per- formed on the dataset obtained by the GeneChip technology (Microarray Suite 4). The images were scaled to a median signal intensity of 200. All datasets remained otherwise unmodified, except where mentioned in the text. False change rates and significance tests were additionally carried out with GeneSpring ${ }^{\circledR}$ (Silicon Genetics, Redwood City, CA, USA) after conversion of the file data by Microarray Suite 5 .

Microarray data from human renal biopsy RNA were analyzed using GeneSpring. Only genes with expression levels above 100 in two of four samples from each group and amplification were analyzed. Analysis was performed as described in the text.

\section{RESULTS}

Aliquots of total RNA from human PBMCs and HK cells were amplified once, twice, or three times in duplicate, starting from $1 \mu \mathrm{g}, 100 \mathrm{ng}$, and $1 \mathrm{ng}$ RNA, respectively. The quality of the biotinylated amplified aRNA was examined by size distribution with an Agilent 2100 Bioanalyzer. As reported for different methods of linear RNA amplification, the average size distribution of the aRNA is similar to the size of the original RNA population during the first round of amplification, but it decreases with each additional round (Figure 1, A-D) $(2,13)$. The aRNA was hybridized to Affymetrix HG-U95Av2 microarrays.

A prerequisite for studying the reproducibility of the amplification method is to assess the bias introduced by the hybridization procedure and the Affymetrix system themselves. The reproducibility of the system was quantified by the determining the Pearson correlation coefficient $r$ between duplicate hybridizations of two aliquots of PBMC RNA. The values of 0.996 and 0.994 were very similar to those obtained in hybridizations of duplicate aRNAs after a single round of amplification $(1 \times)(r=0.997$ for PBMC $1 \times$, and $r=0.993$ for $\mathrm{HK}$ $1 \times)$ and after two rounds of amplification $(2 \times)(r=$ 0.988 for PBMC $2 \times, r=$ 0.992 for $\mathrm{HK} 2 \times$ ), suggesting a high reproducibility of the protocol (Figure 2A, a-c). Even an additional third round of amplification had only a modest effect on reproducibility, as indicated by the high correlation coefficients $(r=0.984$ for PBMC $3 \times, r=0.970$ for HK $3 \times)$. These high values suggested a reproducibility of the bias introduced by both the Affymetrix system and the amplification protocol.

To measure the bias caused by using different amounts of starting material, we calculated the correlation coefficients of the datasets obtained after duplicate amplifications of 1 $\mu \mathrm{g}, 100 \mathrm{ng}$, and $1 \mathrm{ng}$ total RNA. We used the notation $(\mathrm{m}(1-1 ; 1-2))$ to denote the
Figure 3. Signal intensities for different probe set categories after multiple rounds of RNA amplification. Probe sets were categorized by their expression level after a single amplification into groups as described in the text. For each microarray, the median expression level of the individual groups was calculated, keeping the probe sets in each group identical. (A) The median ranges from single to double to triple amplification. (B) "Zoom-in" of panel A, to a range up to 10000 (C) Ratios of the median expression levels of each group from different rounds of amplification. (D) Table with all median raw data values for each expression level category. 


\section{Research Report}

median $\mathrm{m}$ of the average difference values between the first single amplification and the second single amplification. The medians of the average difference values of each pair of duplicates were compared to each other. The correlation coefficients measured between successive rounds of amplifications remain fairly constant ( $r=0.937$ and $r=0.934$ ), again indicating a high reproducibility of the procedure (Figure 2A, $\mathrm{d}-\mathrm{f}$ ).

To test how well differences between samples were maintained by the method, we compared the median of two duplicates of PBMC and HK arrays within and across each round of amplification (Figure 2B). Correlation coefficients were largest when samples from the same or consecutive rounds of amplifications were compared and smallest when samples from single and triple amplifications were compared. The correlation coefficient between two datasets of the same amplification round increased slightly in each round of amplification from $r=0.62(1 \times)$ to $r$ $=0.68(3 \times)$. We attribute this phenomenon to a compression of the data range, observing fewer high expression values on successive rounds of amplification (see also Figure 2A) (15).

To investigate the signal enhancement further during the amplification process, we followed the fate of signal intensities of probe set groups during three rounds of amplification. Probe sets were categorized based on their expression levels after a single amplification as follows: probe sets with expression values below 0 , from 0 to 500,500 to 1000,1000 to 2000,2000 to 4000 , 4000 to 6000 , and so on, the last group consisting of probe sets with average difference values higher than 20000 . For each microarray, category, and amplification, the median expression values were calculated, keeping the probe sets in each dataset identical.

We observed a general trend of decrease in expression levels after two and three rounds of amplification, notably more enhanced for high signal intensities (Figure 3, A and B). The ratio of the median expression values of these probe set groups after consecutive rounds of amplification was used as a measure for the enhancement of signal intensities. Again we found that the enhancement of signal intensities was not the same for all ranges of expression levels (Figure 3C). While average difference values were almost identical after one and two rounds of

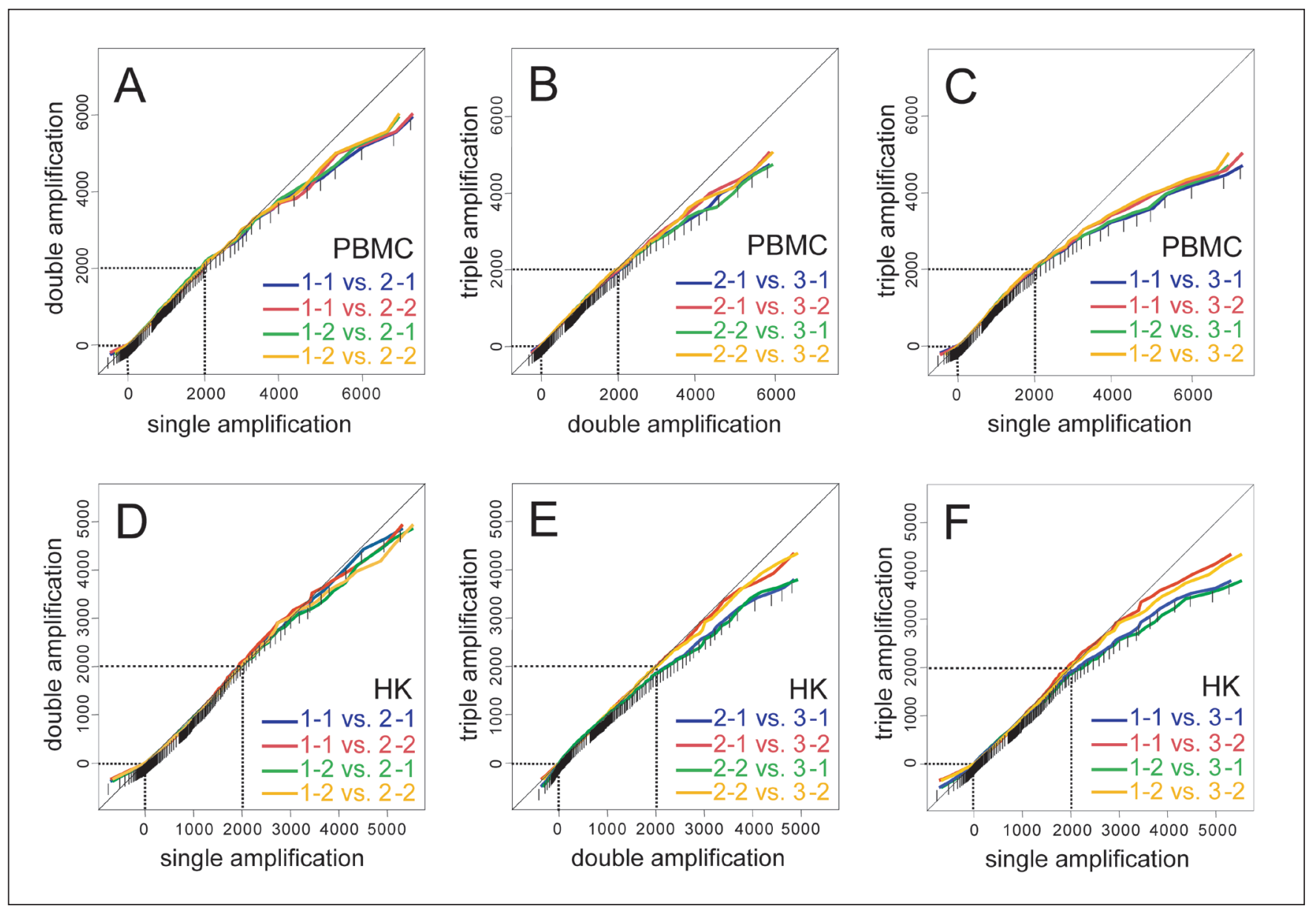

Figure 4. The amplification method is linear for $96 \%$ of the data with positive expression values. For each microarray, the $1 \%, 2 \%$, through $90 \%, 90.1 \%$, $90.2 \%$, through $99 \%$ quantiles were calculated. Comparison of data from single and double amplifications was performed by graphing both single amplification quantiles against both double amplification quantiles, resulting in four polygons. Similar comparisons were done for double-to-triple and single-to-triple amplification data. The proximity of the polygons to the diagonal for expression values between 0 and 2000 indicates that the signal intensities were maintained throughout three rounds of amplification. Signal intensity was reduced for genes with high expression values. Notation of the microarrays is as described in Figure 2. 
amplifications for an expression range roughly between 0 and 4000 [ratio $\mathrm{M}_{2 / 1}\left(\mathrm{~m}_{2 \mathrm{x}} / \mathrm{m}_{1 \mathrm{x}}\right)$ and $\mathrm{M}_{3 / 2}\left(\mathrm{~m}_{3 \mathrm{x}} / \mathrm{m}_{2 \mathrm{x}}\right)=$ 0.8-1.0], it was smaller for other ranges. To investigate this effect in more detail, we performed a quantile analysis on the scaled expression data of all microarrays and all amplifications. For each microarray, we calculated the $1 \%, 2 \%$, through $90 \%, 90.1 \%$, $90.2 \%$, through $99 \%$ quantile.

To compare the data from single and double amplifications, we graphed the single amplification quantiles of both duplicates against both double amplification quantiles, resulting in four polygons (Figure 4, A and D). Similar comparisons were done for double-to-triple and single-to-triple amplification data (Figure 4, B, C, E, and F). While signal intensities above a level of about 2000 are slightly decreasing from amplification to amplification, the curves follow approximately the diagonal (i.e., the identity line) between an expression level of 0 up to about 2000 throughout all comparisons. This indicates that signal intensities in this range are fairly maintained by the amplification method and that the amplification can be assumed almost identical for about $96 \%$ of all 12625 HG-U95Av2 probe sets with expression values above zero.

The question whether similarities and dissimilarities between samples were maintained with multiple rounds of amplification was approached in two ways. First, we inspected how well significant differences between expression profiles of PBMCs and HK cells were conserved throughout the procedure. To that end, we identified differences in gene expression between duplicate samples of PBMCs and HK cells after each round of amplification. Using $\mathrm{S}$ Plus, changes were identified by a rank sum statistics, in which the four expression values of a gene (two for PBMC, two for HK) were ranked, based on signal intensity. If the ranks of the two values in one group (i.e., PBMC or HK) clearly separated from the ranks of the other group, then the gene expression was classified as changed. Comparison of the resulting gene lists in S-Plus and GeneSpring showed that between $75 \%$ and $85 \%$ of all differences were maintained from single to double to triple amplification (data not shown).
To further support our data, we analyzed RNA expression profiles of renal allograft protocol biopsies taken at different stages of posttransplant complications (20). Total RNAs from two independent biopsies (A and $\mathrm{B}$ ) from normal kidneys $(\mathrm{N})$, two biopsies from patients with CTN and two biopsies from patients with CR were amplified in duplicate two and three times, starting from 50 or $1 \mathrm{ng}$ total RNA, respectively $(n=4$ amplifications for each group per round of amplification; Figure 5A). Since only limited amounts of RNA were at our disposition, we could not prepare expression profiles from single rounds of amplification. To quantify the similarity between the groups, the median signal intensities of duplicate amplifications of individual biopsies $(\mathrm{mA}, \mathrm{mB})$ were compared by determining the Pearson correlation coefficient $r$. Duplicates from two biopsies of a group $(\mathrm{mA}, \mathrm{mB})$ were very similar after two amplifications ( $r=$ 0.94-0.99), and this similarity was maintained in a third round, again reflecting the high reproducibility of our procedure (Figure 5B). Dissimilarities between the groups were determined by comparing the Pearson coefficient $r$ for the median $\mathrm{M}$ of $\mathrm{mA}$ and $\mathrm{mB}$ $[\mathrm{M}(\mathrm{mA}, \mathrm{mB})]$ of the groups. As expected from the related nature of $\mathrm{CR}$ and CTN, the correlation coefficient between these groups is higher $(r=0.96)$ than between $\mathrm{N}$ and $\mathrm{CTN}$, or $\mathrm{N}$ and $\mathrm{CR}$ $(r=0.91$ and 0.90 , respectively).

In support of the data of the PBMC and HK comparison (Figure 2B), the correlation coefficients are slightly increased after three rounds of amplification $(r=0.93$ for both $\mathrm{N}$ vs. CTN and $\mathrm{N}$ vs. CR, and $r=0.98$ for CR vs. CTN), indicating a minor compression of the expression values. Strikingly, the bias introduced by the third round of amplification was of the same magnitude for all sample groups: in every case, the correlation coefficients between double-amplified and triple-amplified samples were $r=0.91$.

Plots of raw signal intensities from all 24 microarrays demonstrated a strong conservation of relative expression levels between double and triple amplification, as shown for complement component $\mathrm{C} 1 \mathrm{r}$ with higher-thannormal expression in $\mathrm{CR}$ and $\mathrm{CTN}$, and 


\section{Research Report}

fructose-1,6-biphosphatase (FBP1) with lower-than-normal expression (Figure 5, C and D). Microarray expression data for $\mathrm{C} 1 \mathrm{r}$ and other genes were confirmed by quantitative TaqMan ${ }^{\circledR}$ PCR (data not shown).

\section{DISCUSSION}

In an effort to simplify and further optimize currently available protocols for RNA amplification, we modified the T7 RNA polymerase-based Eberwine protocol (13) by employing commercially available RNA and DNA purification kits and other features. Amplification of $1 \mu \mathrm{g}$ and $100 \mathrm{ng}$ did not substantially introduce more bias than what was introduced by the microarray hybridization procedure itself. Amplification of $1 \mathrm{ng}$ total RNA slightly increased the differences of duplicate samples in all experiments $\left(r_{\text {median }}=\right.$ $0.980, n=8$ ), with an additional increase of the $3^{\prime}: 5^{\prime}$ ratio of the GAPDH and actin control probe sets (data not shown). Loss of 5' complexity, which is a characteristic of all linear amplification methods due to inefficient reverse transcription reaction and use of random primers $(2,11,15)$, has little if any significance, if the microarray probe design takes this into consideration and is $3^{\prime}$ biased. In general, Affymetrix microarray probe sets are directed against 600-nucleotidetarget regions at the very $3^{\prime}$ ends of mRNA (21).

Detailed statistical analysis demonstrated that our method of RNA amplification was highly reproducible and generated almost identical data for about $96 \%$ of probe sets with expression values above zero. Despite minor data compression of expression ranges below zero and above 2000, relative gene expression levels were mostly preserved from one to two and from two to three rounds of amplification (Figure 3). As shown in two independent experiments with different samples, the protocol not only maintained sample similarities but also preserved inter-group dissimilarities (Figures 2B and 5B). For statistical analysis of such data, nonparametric (rank-based) methods are preferred over parametric methods.

We have applied the RNA amplification protocol to monitor differences in gene expression profiles of human renal biopsies with histological evidence of CR or CTN compared to normal samples (N). As part of a detailed data analysis (manuscript in preparation), we found that a large group of genes with significantly higher expression levels in $\mathrm{CR}$ and CTN than in $\mathrm{N}$ were leukocyte specific or complement components (e.g., C1r) (Figure 5C), reminiscent of ongoing minor $\mathrm{T}$ and $\mathrm{B}$ cell infiltration (22). Consistent with decreasing renal function during the development of $\mathrm{CR}$ and CTN, about $50 \%$ of the genes with lower-than-normal expression levels in these samples were genes involved in metabolism (e.g., fructose-1,6-bisphosphatase) (Figure 5D).

Our protocol is applicable to large studies, as it limits the number of handling steps and incorporates commercially available reagents. Instead of performing some reverse transcription reactions in a volume of $1 \mu \mathrm{L}$, which is then lyophilized to $500 \mathrm{~nL}(15)$, we use a constant volume of $20 \mu \mathrm{L}$ throughout all amplifications, which makes handling much easier and requires much less hands-on work and observation. All phenol:chloroform extraction steps, which are typically not consistent and scalable, are omitted. We also deleted the tedious cDNA purification procedure suggested by Baugh et al. (15) and

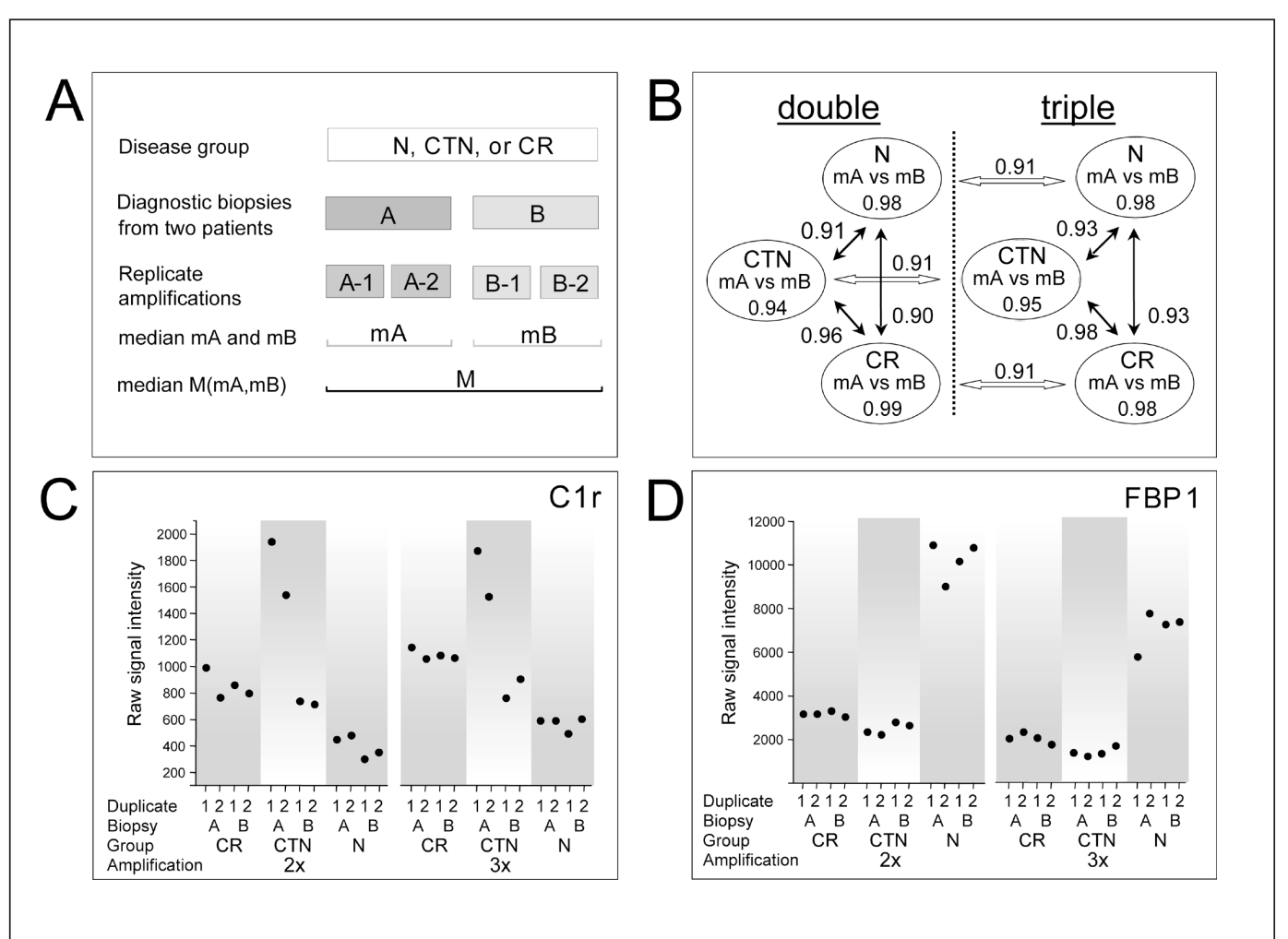

Figure 5. Reproducibility of RNA amplification from clinical samples with different diagnosis. RNA from two individual renal biopsies from healthy $(\mathrm{N}), \mathrm{CTN}$, and CR kidneys were amplified in duplicatesfrom $50 \mathrm{or} 1 \mathrm{ng}$. The Pearson correlation coefficent $r$ of the RNA expression values was calculated for the median $\mathrm{m}$ of the duplicate groups. The inter-group differences are conserved after three rounds of amplification. (A) Design of the experiment and the analysis. (B) Correlation coefficients of the individual comparisons based on the raw expression values. The numbers are the Pearson correlation coefficients $r$. $\mathrm{mA}$ and $\mathrm{mB}$, median expression values of the duplicates from biopsy A and biopsy $\mathrm{B}$, respectively. (C and D) Plot of 24 average difference values for $\mathrm{C} 1 \mathrm{r}$ (complement component 1r; GenBank ${ }^{\circledR}$ accession no. M14018; panel C) and fructose-1,6-biphosphatase 1 (FBP1; GenBank accession no. U21931; panel D). 
instead introduced QIAquick cDNA purification columns, which completely removed the T7/oligo-dT primer from the reaction mixture (data not shown). This step eliminates the carryover of the primer into the in vitro transcription reaction and thus helps to increase the mean average difference after GeneChip hybridization (15).

The volumes of DNA- or RNA-containing column eluates are narrowed down under vacuum, until dryness is reached. This step omits tedious observation of the volume and additional volume adjustments. Our protocol modifications do not compromise the reproducibility of the results, which are very well comparable with those from other linear amplification protocols. Specifically, the correlation coefficients for replicates of single and double amplifications ( $r=0.995, r=0.988$, respectively) are similar to those of Baugh's protocol $(r=0.992 ; r=0.986)$ (15); the correlation coefficient between single and double amplification are about the same in both procedures $[r=0.94(n=10$; Reference 15) and $r=$ $0.93(n=8)]$. The degree of data compression $[\Delta r=0.02$ (Reference 15) vs. 0.014-0.027 (this work)] and the maintenance of changes in gene expression $(75 \%-80 \%)$ are also comparable in both protocols (15).
In conclusion, our analysis shows that the modified Eberwine protocol as described here offers simplified features that improve sample handling and provide high reproducibility of microarray data obtained from amplification of as little as $1 \mathrm{ng}$ total RNA. The data presented here suggest that this protocol will prove to be a valuable tool for a wide range of RNA expression profiling applications.

\section{ACKNOWLEDGMENTS}

We would like to thank Dr. John Hogenesch for valuable discussions and reviewing the manuscript. We thank Dr. Jordi Bover for providing the biopsy RNA samples.

\section{REFERENCES}

1.Lockhart, D.J. and E.A. Winzeler. 2000. Genomics, gene expression and DNA arrays. $\mathrm{Na}-$ ture 405:827-833.

2.Eberwine, J.H., H. Yeh, K. Miyashiro, Y. Cao, S. Nair, R. Finnell, M. Zettel, and P. Coleman. 1992. Analysis of gene expression in single live neurons. Proc. Natl. Acad. Sci. USA 89:3010-3014.

3.Eberwine, J.H., J.E. Kacharmina, C. Andrews, K. Miyashiro, T. McIntosh, K. Becker, T. Barrett, D. Hinkle, et al. 2001. mRNA expression analysis of tissue sections and single cells. J. Neurosci. 21:8310-8314.

4.Welsh, J.B., L.M. Sapinoso, A.I. Su, S.G. Kern, J. Wang-Rodriguez, C.A. Moskaluk, H.F. Frierson, Jr., and G.M. Hampton. 2001. Analysis of gene expression identifies candidate markers and pharmacological targets in prostate cancer. Cancer Res. 61:59745978.

5.Luo, L., R.C. Salunga, H. Guo, A. Bittner, K.C. Joy, J.E. Galindo, H. Xiao, K.E. Rogers, et al. 1999. Gene expression profiles of laser-captured adjacent neuronal subtypes. Nat. Med. 5:117-122.

6.Mariani, T.J., J.J. Reed, and S.D. Shapiro. 2002. Expression profiling of the developing mouse lung: insights into the establishment of the extracellular matrix. Am. J. Respir. Cell Mol. Biol. 26:541-548.

7.Panda, S., J.B. Hogenesch, and S.A. Kay. 2002. Circadian rhythm from flies to human. Nature 417:329-335.

8.Meltzer, P.S. 2001. Spotting the target: microarrays for disease gene discovery. Curr. Opin. Gen. Dev. 11:258-263.

9.Orr, M.S. and U. Scherf. 2002. Large-scale gene expression analysis in molecular target discovery. Leukemia 16:473-477.

10.Affymetrix. 2001. Affymetrix GeneChip ${ }^{\circledR}$ Expression Analysis Technical Protocol. Affymetrix, Palo Alto, CA.

11.Kacharmina, J.E., P.B. Crino, and L.H.
Eberwine. 1999. Preparation of cDNA from single cells and subcellular regions. Methods Enzymol. 303:3-18.

12.Saiki, R., T. Bugawan, G. Horn, K. Mullis, and H. Erlich. 1986. Analysis of enzymatically amplified $\beta$-globin and HLA-DQ $\alpha$ DNA with allele-specific oligonucleotide probes. Nature 324:163.

13.Van Gelder, R.N., M.E. von Zastrow, A. Yool, W.C. Dement, J.D. Barchas, and J.H. Eberwine. 1990. Amplified RNA synthesized from limited quantities of heterogeneous cDNA. Proc. Natl. Acad. Sci. USA 87:16631667.

14.Jeffreys, A., V. Wilson, R. Neumann, and J. Keyte. 1988. Amplification of human minisatellites by the polymerase chain reaction: towards DNA fingerprinting of single cells. Nucleic Acids Res. 16:10953-10971.

15.Baugh, L.R., E.L. Hill, and C.P. Hunter. 2001. Quantitative analysis of mRNA amplification by in vitro transcription. Nucleic Acids Res. 29:e29.

16.Wang, E., L.D. Miller, G.A. Ohnmacht, E.T Liu, and F.M. Marincola. 2000. High-fidelity mRNA amplification for gene profiling Nat. Biotechnol. 18:457-459.

17.Pabón, C., Z. Modrusan, M.V. Ruvolo, I.M. Coleman, S. Daniel, H. Yue, L.J. Arnold, Jr., and M.A. Reynolds. 2001. Optimized T7 amplification system for microarray analysis. BioTechniques 31:874-879.

18.Serón, D., F. Moreso, J. Bover, E. Condom, S. Gil-Vernet, C. Canas, X. Fulladosa, J. Torras, et al. 1997. Early protocol allograft biopsies and graft outcome. Kidney Int. 51:310-317.

19.Racusen, L.C., K. Solez, R.B. Colvin, S.M. Bonsib, M.C. Castro, T. Cavallo, B.P. Croker, A.J. Demetris, et al. 1999. The BANFF 97 working classification of renal allograft pathology. Kidney Int. 55:713-723.

20.Jindal, R.M. and S. Hariharan. 1999. Chronic rejection in kidney transplants: an indepth review. Nephron 83:13-24.

21.Affymetrix. 2001. Affymetrix Technical Note: Array Design for the GeneChip ${ }^{\circledR} \mathrm{Hu}-$ man Genome U133 Set. Affymetrix, Palo Alto, CA.

22.Kinoshita, T. 1991. Biology of complement: the overture. Immunol. Today 12:291-295.

Received 18 July 2002; accepted 17 December 2002.

Address correspondence to:

Dr. Andreas Scherer

Transplantation BU/Research

WSJ-386.6.31

CH-4002 Basel, Switzerland

e-mail: andreas.scherer@pharma.novartis.com 